\title{
CTLA4 promoter methylation predicts response and progression-free survival in stage IV melanoma treated with anti-CTLA-4 immunotherapy (ipilimumab)
}

\author{
Simon Fietz ${ }^{1,2} \cdot$ Romina Zarbl $^{2}$. Dennis Niebel ${ }^{1}$. Christian Posch ${ }^{3,4}$. Peter Brossart ${ }^{5}$. Gerrit H. Gielen ${ }^{6}$. \\ Sebastian Strieth ${ }^{2} \cdot$ Torsten Pietsch $^{6}$. Glen Kristiansen ${ }^{7}$. Friedrich Bootz ${ }^{2}$. Jennifer Landsberg ${ }^{1} \cdot$ Dimo Dietrich $^{2}$ D
}

Received: 23 July 2020 / Accepted: 20 October 2020 / Published online: 16 November 2020

(c) The Author(s) 2020

\begin{abstract}
Anti-CTLA-4-antibodies can induce long-lasting tumor remissions. However, only a few patients respond, necessitating the development of predictive companion biomarkers. Increasing evidence suggests a major role of epigenetics, including DNA methylation, in immunology and resistance to immune checkpoint blockade. Here, we tested CTLA4 promoter methylation and CTLA-4 protein expression as predictive biomarkers for response to anti-CTLA-4 immunotherapy. We identified retrospectively $N=30$ stage IV melanoma patients treated with single-agent anti-CTLA-4 immunotherapy (ipilimumab). We used quantitative methylation-specific PCR and immunohistochemistry to quantify CTLA4 methylation and protein expression in pre-treatment samples. CTLA4 methylation was significantly higher in progressive as compared to responding tumors and significantly associated with progression-free survival. A subset of infiltrating lymphocytes and tumor cells highly expressed CTLA-4. However, CTLA-4 protein expression did not predict response to treatment. We conclude that CTLA4 methylation is a predictive biomarker for response to anti-CTLA-4 immunotherapy.
\end{abstract}

Keywords CTLA-4 $\cdot$ CTLA4 $\cdot$ DNA methylation $\cdot$ Immunotherapy $\cdot$ Predictive biomarker $\cdot$ Melanoma

Jennifer Landsberg and Dimo Dietrich are the joint senior authors in this work.

\section{Dimo Dietrich}

dimo.dietrich@gmail.com

1 Department of Dermatology and Allergy, University Hospital Bonn, Venusberg-Campus, Bonn 53127, Germany

2 Department of Otolaryngology, Head and Neck Surgery, University Hospital Bonn, Venusberg-Campus, 53127 Bonn, Germany

3 Department of Dermatology and Allergy, Technical University of Munich, Munich, Germany

4 Faculty of Medicine, Sigmund Freud University, Vienna, Austria

5 Department of Oncology, Hematology and Rheumatology, University Hospital Bonn, Bonn, Germany

6 Institute of Neuropathology, University Hospital Bonn, Bonn, Germany

7 Institute of Pathology, University Hospital Bonn, Bonn, Germany

\section{Introduction}

Therapeutic monoclonal antibodies (mAbs) targeting the immune checkpoints cytotoxic $\mathrm{T}$ lymphocyte-associated protein 4 (CTLA-4) and programmed cell death 1 (PD-1) have revolutionized the treatment of various cancers. In metastatic melanoma immune checkpoint blockade (ICB) considerably prolongates survival and even leads to durable remission in some cases [1, 2]. However, only a subgroup of patients responds to treatment due to primary resistance to ICB. This is particularly true for ipilimumab, the first in class CTLA-4-directed immune checkpoint inhibitor, which has shown efficacy in $19 \%$ of patients [2]. Compared to anti-PD-1 inhibitors, e.g., nivolumab and pembrolizumab, the efficacy of ipilimumab is lower and accompanied with $30 \%$ more high-grade side effects than nivolumab [2]. Since a small group of patients shows dramatic responses to ipilimumab monotherapy, CTLA-4 remains an important immunotherapy target. Hence, robust biomarkers indicating patients, who benefit from anti-CTLA-4 first-line monotherapy, are needed in the field of personalized medicine. Such biomarkers are of considerable interest since next-generation 
CTLA-4-directed compounds (mono- or bispecific antibodies, probodies) are under investigation in preclinical up to phase III trials. According to preclinical trials, ipilimumab probodies are believed to show comparable efficacy with less adverse reactions [3]. Therefore, it constitutes a promising companion drug in the treatment of advanced melanoma. Ongoing biomarker research mainly focuses on response data of patients treated with anti-PD-1 mAbs [4]. Of particular interest are parameters of the tumor microenvironment, such as tumor-infiltrating immune cells (TILs), immune checkpoint (mainly PD-L1) expression, and immune gene signatures [5]. However, genetic alterations, for instance, microsatellite instability (MSI), mutational and neoantigen burden are also actively investigated [6, 7]. Epigenetic alterations, however, have widely been neglected as potential predictive biomarkers so far but have great potential due to their significance in tumorigenesis and immunology, including immune cell differentiation and $\mathrm{T}$ cell exhaustion [8-12]. Recent reports suggest DNA methylation of genes encoding for the immune checkpoints 4-1BB, LAG-3, PD-L2, and CTLA-4 as predictive biomarkers for response to ICB in melanoma [13-16]. Our present study aimed at the analysis of the value of CTLA4 methylation as a biomarker to predict response and progression under CTLA-4-directed ICB monotherapy. In addition, we compared CTLA4 methylation with CTLA-4 protein expression as a potential predictive biomarker.

\section{Materials and methods}

Our study comprised histologically confirmed pretreatment melanomas from a cohort of $N=30$ stage IV melanoma patients treated with anti-CTLA-4 antibody ipilimumab as first-line immunotherapy at the University Hospital Bonn and Technical University of Munich between 2010 and 2015. The patients received $2-6$ doses ( $3 \mathrm{mg} / \mathrm{kg}$ body weight) ipilimumab. Median number of applications was four. Our study was approved by the Institutional Review Board (IRB) of the University Hospital Bonn (vote no. 187/16). Patient and sample characteristics are described in detail in Table 1. We analyzed PFS and response as clinical endpoint. PFS was defined as time between the first application of anti-CTLA-4 ICB and the date of documented disease progress or last contact, respectively. Response was determined in accordance with RECIST version 1.1.

For methylation analysis, we used FFPE tumor tissues mounted on glass slides. After tumor macrodissection, we applied the innuCONVERT Bisulfite All-In-One Kit (Analytik Jena, Jena, Germany) for tissue lysis, bisulfite conversion, and DNA purification following the manufacturer's protocol. We performed qMSP to evaluate CTLA4 promoter methylation as previously described [16]. In brief, we determined relative CTLA4 methylation levels referred to total DNA in duplex qMSP reactions. A CpG-free target region within the housekeeping gene $A C T B$ was used as reference.

Prior to immunohistochemistry, 4- $\mu \mathrm{m}$ FFPE tumor tissues sections were deparaffinizated with xylene, rehydrated through a descending ethanol series, and finally washed with $550 \mathrm{mM}$ Tris-buffered saline (TBS). Heavily pigmented melanoma sections were bleached with $30 \% \mathrm{H}_{2} \mathrm{O}_{2}$ and $0.5 \%$ potassium hydroxide for $30 \mathrm{~min}$ at $37^{\circ} \mathrm{C}$. For antigen retrieval, the sections were incubated with Target Retrieval Solution (pH6, Dako/ Agilent Technologies, Inc., Santa Clara, CA, USA) at $100{ }^{\circ} \mathrm{C}$ for $10 \mathrm{~min}$ and were washed with TBS subsequently. Primary CTLA- 4 antibody (dilution 1:50, mouse monoclonal antibody, clone: BSB-88, Bio $\mathrm{SB}$, Santa Barbara, CA, USA) was added, incubated at $4{ }^{\circ} \mathrm{C}$ overnight, and subsequently washed with $550 \mathrm{mM}$ TBS. REAL Detection System Alkaline Phosphatase/RED (Dako/ Agilent Technologies) was utilized to visualize bounded primary antibody according to the manufacturer's protocol. Finally, we used Mayer's Hemalum solution (Merck Millipore, Billerica, MA, USA) to contrast the staining. CTLA-4 expression by tumor cells was quantified using the $\mathrm{H}$ scoring system [17]. Tumor-infiltrating lymphocytes were assessed using the scoring system by Clark: absent $=$ no TILs, nonbrisk $=$ focal TILs, brisk $=$ diffuse TILs [18]. CTLA-4-expressing immune cells were analyzed qualitatively.

Statistical tests were performed utilizing SPSS, version 23.0 (SPSS Inc., Chicago, IL). Kaplan-Meier and Cox proportional hazards regression analyses were conducted ( $P$ values refer to log-rank and Wald tests, respectively). Survival analyses were performed using dichotomized methylation levels and categorized variates, respectively. Mann-Whitney $U$ test (2 groups) and Kruskal-Wallis ( $>2$ groups) test were applied for arithmetical mean comparison. Correlations were computed using Spearman's rank correlation (Spearman's $\rho$ ). Two-sided $P$ values $<0.05$ were considered statistically significant.

\section{Results}

\section{Analyses of CTLA4 methylation in $N=30 \mathrm{FFPE}$ melanoma samples from patients prior ipilimumab therapy}

To test the utility of CTLA4 methylation as a predictive biomarker for response to anti-CTLA-4 immune checkpoint blockade in stage IV melanoma patients, we identified retrospectively 30 patients diagnosed with advanced melanoma and treated with anti-CTLA-4 antibody ipilimumab monotherapy. Median time from biopsy to initiation of antiCTLA-4 blockade was 7 months. Best objective response to anti-CLTA-4 therapy using the Response Evaluation 
Table 1 Patient characteristics at baseline and their associations with PFS, response, and CTLA4 promoter methylation

\begin{tabular}{|c|c|c|c|c|c|c|c|}
\hline \multirow[t]{2}{*}{ Characteristic } & \multirow[t]{2}{*}{ Total cohort $(N=30)$} & \multicolumn{2}{|l|}{ PFS } & \multicolumn{2}{|c|}{ CTLA4 methylation } & \multicolumn{2}{|l|}{ Response } \\
\hline & & Hazard ratio $[95 \% \mathrm{CI}]$ & $P$ value & Mean $(\%),[S D]$ & $P$ value & Responder $(N=6)$ & $\begin{array}{l}\text { Non- } \\
\text { responder } \\
(N=24)\end{array}$ \\
\hline $\begin{array}{l}\text { Median age (range)- } \\
\text { years }\end{array}$ & $64(28-86)$ & & & & & $65(49-76)$ & $64(28-86)$ \\
\hline Sex-no. $(\%)$ & & & & & $P=0.54$ & & \\
\hline Male & $17(57)$ & Ref group & & $22.8[30.5]$ & & $5(83)$ & $12(50)$ \\
\hline Female & $13(43)$ & 2.58 [1.13-5.87] & $P=0.024$ & 17.5 [19.7] & & $1(17)$ & $12(50)$ \\
\hline $\begin{array}{l}\text { Disease origin-no. } \\
(\%)\end{array}$ & & & $P=0.13$ & & $P=0.13$ & & \\
\hline Cutaneous & $20(67)$ & Ref group & & $17.2[18.1]$ & & $4(67)$ & $16(67)$ \\
\hline Acral & $2(7)$ & $1.67[0.37-7.54]$ & $P=0.50$ & $23.2[3.9]$ & & $0(0)$ & $2(8)$ \\
\hline Uveal & $1(3)$ & $1.01[0.13-7.87]$ & $P=0.99$ & 24.7 & & $0(0)$ & $1(4)$ \\
\hline Meningeal & $1(3)$ & $0.69[0.09-5.11]$ & $P=0.70$ & 130.4 & & $0(0)$ & $1(4)$ \\
\hline $\begin{array}{l}\text { Melanoma of unknown } \\
\text { primary (MUP) }\end{array}$ & $2(7)$ & $11.2[1.81-69.2]$ & $P=0.009$ & $7.8[6.7]$ & & $0(0)$ & $2(8)$ \\
\hline Not available & $4(13)$ & NA & NA & NA & & $2(33)$ & $2(8)$ \\
\hline $\begin{array}{l}\text { Stage at baseline-no. } \\
(\%)\end{array}$ & & & $P=0.59$ & & $P=0.79$ & & \\
\hline M1a & $3(10)$ & $2.49[0.60-10.3]$ & $P=0.21$ & $11.9[8.7]$ & & $0(0)$ & $3(13)$ \\
\hline M1b & $3(10)$ & $1.57[0.41-6.11]$ & $P=0.51$ & $16.2[8.7]$ & & $1(17)$ & $2(8)$ \\
\hline M1c & $13(43)$ & $1.74[0.65-4.69]$ & $P=0.27$ & $21.6[21.7]$ & & $1(17)$ & $12(50)$ \\
\hline M1d & $10(33)$ & Ref group & & $21.6[38.4]$ & & $4(67)$ & $6(25)$ \\
\hline Unknown & $1(3)$ & NA & NA & NA & & $0(0)$ & $1(4)$ \\
\hline $\begin{array}{l}\text { Brain metastases at } \\
\text { baseline- no. }(\%)\end{array}$ & & & & & $P=0.46$ & & \\
\hline No & $19(63)$ & Ref group & & $19.2[18.6]$ & & $2(33)$ & $17(71)$ \\
\hline Yes & $10(33)$ & $0.56[0.23-1.40]$ & $P=0.22$ & $21.6[38.4]$ & & $4(67)$ & $6(25)$ \\
\hline Unknown & $1(3)$ & NA & NA & NA & & $0(0)$ & $1(4)$ \\
\hline $\begin{array}{l}\text { BRAF mutation-no. } \\
(\%)\end{array}$ & & & & & $P=0.22$ & & \\
\hline Yes & $5(17)$ & 5.15 [1.43-18.5] & $P=0.012$ & $17.8[9.1]$ & & $0(0)$ & $5(21)$ \\
\hline No & $23(77)$ & Ref group & & $21.4[29.5]$ & & $5(83)$ & $18(75)$ \\
\hline Not available & $2(7)$ & NA & NA & NA & & $1(17)$ & $1(4)$ \\
\hline $\begin{array}{l}\text { NRAS mutation-no. } \\
(\%)\end{array}$ & & & & & $P=0.24$ & & \\
\hline Yes & $14(47)$ & $0.98[0.97-1.00]$ & $P=0.97$ & $13.7[12.0]$ & & $3(50)$ & $11(46)$ \\
\hline No & $6(20)$ & Ref group & & $27.9[27.0]$ & & $1(17)$ & $5(21)$ \\
\hline Not available & $10(33)$ & NA & NA & NA & & $2(33)$ & $8(33)$ \\
\hline $\begin{array}{l}\text { LDH at baseline-no. } \\
(\%)\end{array}$ & & & & & $P=0.72$ & & \\
\hline Normal & $16(53)$ & Ref group & & $20.6[31.4]$ & & $5(83)$ & $11(46)$ \\
\hline Elevated & $11(37)$ & $3.08[1.14-8.30]$ & $P=0.026$ & 18.3 [20.2] & & $0(0)$ & $11(46)$ \\
\hline Not available & $3(10)$ & NA & NA & NA & & $1(17)$ & $2(8)$ \\
\hline $\begin{array}{l}\text { Previous therapies- } \\
\text { no. }(\%)^{\S}\end{array}$ & & & $P=0.59$ & & $P=0.46$ & & \\
\hline Chemotherapy & $7(23)$ & $0.86[0.35-2.24]$ & $P=0.80$ & 21.5 [27.6] & & $1(17)$ & $6(25)$ \\
\hline Targeted therapy & $2(7)$ & $2.07[0.46-9.31]$ & $P=0.34$ & $9.7[1.6]$ & & $0(0)$ & $2(8)$ \\
\hline None & $21(70)$ & Ref group & & $20.6[27.8]$ & & $5(83)$ & $16(67)$ \\
\hline $\begin{array}{l}\text { Sample origin-no. } \\
(\%)\end{array}$ & & & $P=0.65$ & & $P=0.48$ & & \\
\hline Skin & $10(33)$ & Ref group & & 23.9 [22.9] & & $1(17)$ & $9(38)$ \\
\hline
\end{tabular}


Table 1 (continued)

\begin{tabular}{|c|c|c|c|c|c|c|c|}
\hline \multirow[t]{2}{*}{ Characteristic } & \multirow[t]{2}{*}{ Total cohort $(N=30)$} & \multicolumn{2}{|l|}{ PFS } & \multicolumn{2}{|c|}{ CTLA4 methylation } & \multicolumn{2}{|l|}{ Response } \\
\hline & & Hazard ratio $[95 \% \mathrm{CI}]$ & $P$ value & Mean (\%), [SD] & $P$ value & Responder $(N=6)$ & $\begin{array}{l}\text { Non- } \\
\text { responder } \\
(N=24)\end{array}$ \\
\hline Lymph node & $10(33)$ & $0.69[0.26-1.81]$ & $P=0.45$ & $11.1[8.0]$ & & $2(33)$ & $8(33)$ \\
\hline Lung & $4(13)$ & 0.63 [0.19-2.09] & $P=0.45$ & $9.5[3.6]$ & & $1(17)$ & $3(13)$ \\
\hline Liver & $2(7)$ & $0.82[0.17-3.89]$ & $P=0.80$ & $26.3[22.3]$ & & $0(0)$ & $2(8)$ \\
\hline Brain & $3(10)$ & $0.24[0.05-1.14]$ & $P=0.073$ & $49.9[69.7]$ & & $2(33)$ & $1(4)$ \\
\hline Uvea & $1(3)$ & $0.74[0.09-5.98]$ & $P=0.78$ & 24.7 & & $0(0)$ & $1(4)$ \\
\hline Response-no. (\%) & & & ND & & $P=0.052$ & & \\
\hline Objective response $\mathrm{e}^{¥}$ & $6(20)$ & ND & ND & $7.6[2.3]$ & & $6(100)$ & $0(0)$ \\
\hline Progressive disease & $21(70)$ & Ref group & & $23.7[29.9]$ & & $0(0)$ & $21(86)$ \\
\hline Stable disease & $3(10)$ & $0.30[0.07-1.33]$ & $P=0.11$ & $24.6[16.1]$ & & $0(0)$ & $3(13)$ \\
\hline TILs-no. $(\%)$ & & & & & $P=0.086$ & & \\
\hline Non-brisk/brisk & $20(67)$ & $0.79[0.36-1.76]$ & $P=0.57$ & $11.7[6.2]$ & & $4(67)$ & $16(67)$ \\
\hline Absent & $10(33)$ & Ref group & & $38.1[39.9]$ & & $2(33)$ & $8(33)$ \\
\hline $\begin{array}{c}\text { CTLA-4 }{ }^{+} \text {tumor } \\
\text { cells-no. }(\%)\end{array}$ & & & $P=0.24$ & & $P=0.29$ & & \\
\hline H score $\geq 200$ & $6(20)$ & $1.73[0.63-4.73]$ & $P=0.28$ & $17.5[11.2]$ & & $1(17)$ & $5(21)$ \\
\hline H score100-199 & $10(33$ & $2.17[0.85-5.50]$ & $P=0.10$ & $23.0[22.4]$ & & $1(17)$ & $9(38)$ \\
\hline H score $\leq 99$ & $14(47)$ & Ref group & & $20.0[33.3]$ & & $4(67)$ & $10(42)$ \\
\hline
\end{tabular}

NA not analyzed

${ }^{\S}$ Previous therapies included chemotherapy and targeted therapy

${ }^{\ddagger}$ This category included patients with a complete response $(N=3)$ and those with a partial response $(N=3)$

$N D$ not determined (hazard ratio and $P$ values could not be calculated because of complete separation of the predictor variable CR/PR)

Criteria in Solid Tumors (RECIST) version 1.1 included $3 / 30$ (10\%) with complete response (CR), 3/30 (10\%) with partial response (PR), 3/30 (10\%) with stable disease (SD), and 21/30 (70\%) with progressive disease (PD). All patients that achieved complete response had brain metastases and received ipilimumab without radiation therapy. Two of these patients remained tumor-free (5.3 years and 5.9 years followup time). Overall response rate was $20 \%$ (CR + PR). 9/30 (30\%) patients were previously treated with targeted therapy or chemotherapy, whereas $21 / 30(70 \%)$ were therapy naïve. Median follow-up for survival was 3 months. Clinical characteristics are described in detail in Table 1. We performed a quantitative methylation-specific PCR assay (qMSP) targeting the CpG-site of interest using DNA from formalin-fixed and paraffin-embedded (FFPE) melanoma samples prior ipilimumab treatment.

\section{Association of CTLA4 methylation with response to ipilimumab and PFS}

First, we tested the association of CTLA4 promoter methylation with response according to RECIST version 1.1. Despite the small sample size, we could find significantly lower methylation levels in melanoma samples from responders [complete and partial responders; mean methylation level $7.6 \pm 2.3,6 / 30(20 \%)]$ compared to patients with progressive disease (mean methylation level 23.7 $\pm 29.9,21 / 30$ (70\%), $P=0.042$ ) and to patients with stable disease (mean methylation level $24.4 \pm 16.1,3 / 30$ (10\%), $P=0.024$, Fig. 1a). We did not find significant methylation differences between samples from patients with stable disease compared to patients with progressive disease.

We further tested the association of CTLA4 promoter methylation with progression-free survival (PFS). We classified patient samples according to the median methylation (10.9\%) of the whole cohort as CTLA4 methylation above median and below median, respectively. We used median methylation as cutoff for dichotomization of methylation levels to avoid an overfitted model due to the introduction of an optimized cutoff. Patients with tumor methylation levels below median showed a significantly better PFS as compared to patients with methylation levels above cutoff $(P=0.014$, Fig. 1b). Patients with low methylated tumors had a PFS of $33 \%$ after 1 year ( $40 \%$ after 6 months and $13 \%$ after 5 years) while all patients with highly methylated melanomas progressed within 6 months after immunotherapy start. 


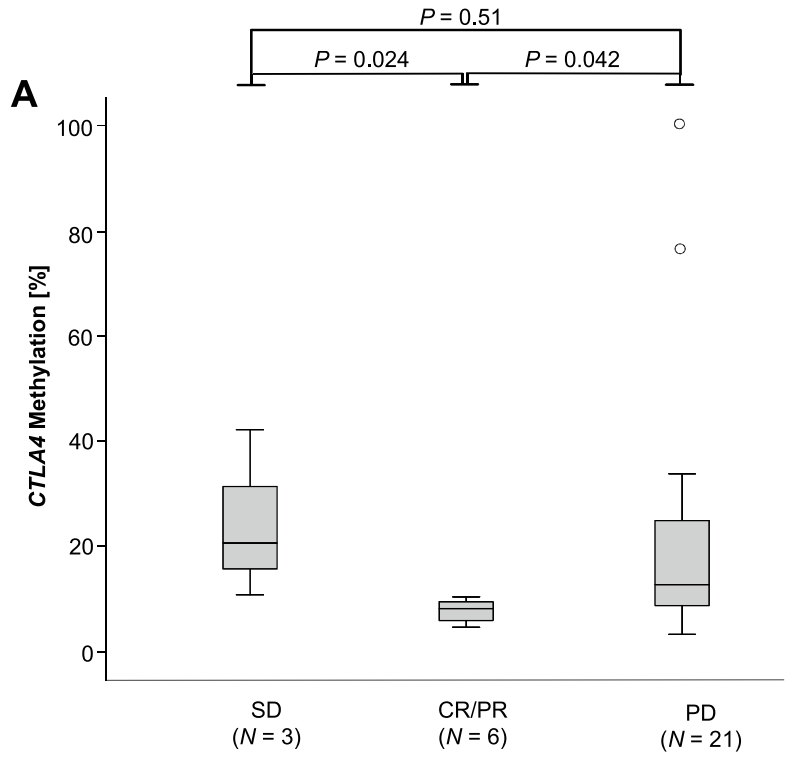

Response to Anti-CTLA-4 Treatment (Ipilimumab)

Fig. 1 Association of CTLA4 promoter methylation with response and PFS in melanoma patients treated with ipilimumab. a CTLA4 methylation levels in melanomas from patients with stable disease

\section{Association of clinical, molecular, and histomorphological features with PFS and CTLA4 methylation}

Significantly worse PFS was associated with female sex, $B R A F$-mutated tumors, and patients with increased LDH levels at baseline (Table 1). We found no significant association between TILs and PFS or response to ipilimumab. However, we found a trend towards lower methylation levels in melanomas with brisk/non-brisk TILs (mean methylation level $11.7 \pm 6.2,20 / 30(67 \%)$ ) compared to melanomas with no TILs (mean methylation level 38.1 $\pm 39.9,10 / 30$ (33\%), $P=0.086$, Table 1$)$.

\section{Association of CTLA4 methylation and CTLA-4 protein expression}

We have previously shown that CTLA4 promoter methylation inversely correlates with its corresponding mRNA expression (Spearman's $\rho=-0.42, P<0.001$ ) in a large melanoma cohort from The Cancer Genome Atlas [16]. Following up on this finding and to dissect melanoma or immune cells as source of CTLA-4 expression, we performed IHC for CTLA-4 protein expression in our melanoma cohort prior ipilimumab treatment. As expected, positive CTLA-4 IHC staining was found for a subset of lymphocytes in tonsillar tissue used as a positive control (Fig. 2a). In melanomas, we found CTLA-4 protein expressed predominantly on tumor cells and only on a small subset of infiltrating

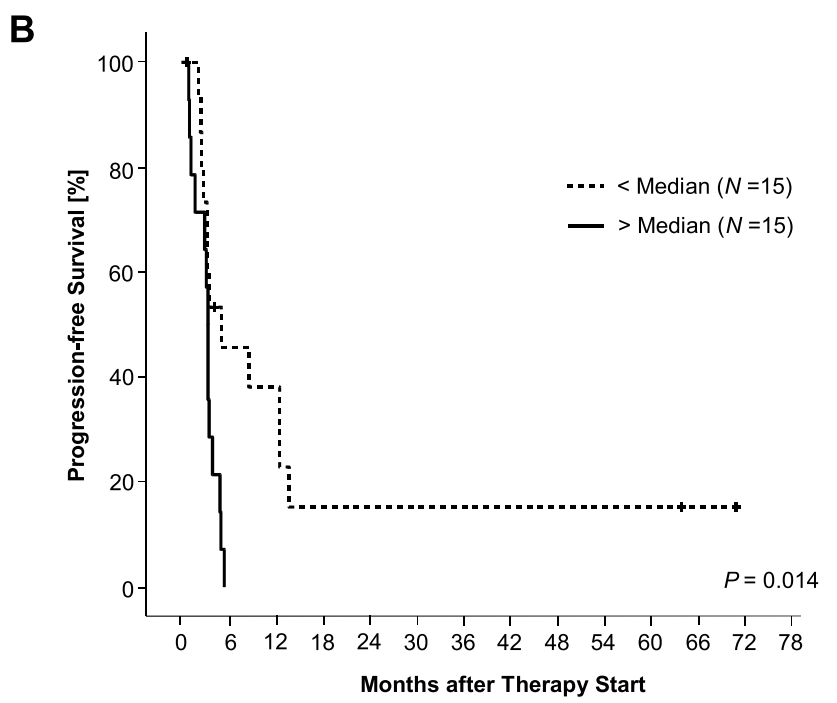

(SD), complete or partial response (CR/PR), and progressive disease (PD). b Kaplan-Meier analyses of PFS in melanoma patients receiving anti-CTLA-4 monotherapy (ipilimumab)

immune cells (Fig. 2b). CTLA-4 expression by melanoma cells was heterogeneous, e.g., tumors with predominantly CTLA-4-negative tumor cells, tumors with mainly weakly CTLA-4-expressing tumor cells, and melanomas with strongly CTLA-4-expressing tumor cells (Fig. 2c). Quantitative CTLA-4 protein expression as determined using the $\mathrm{H}$ scoring system did not reveal a significant correlation between methylation and protein expression (Spearman's $\rho=0.21, P=0.27$ ). Only a subset of tumors contained CTLA $-4^{+}$immune cells $(40 \%, 12 / 30)$. We could not find a correlation between CTLA4 methylation and the presence of CTLA $-4^{+}$immune cells $(P=0.91)$. In concordance with the lack of a significant correlation between methylation and protein expression, we did not find significant differences in Kaplan-Meier analysis of PFS in our cohort stratified by CTLA-4 protein-expressing tumor cells $(P=0.17)$ or TILs $(P=0.26)$, respectively.

\section{Discussion}

We have previously reported the utility of CTLA4 promoter methylation as a predictive biomarker for response to immunotherapy and survival in a heterogeneous cohort comprised of $N=50$ melanoma patients who received anti-PD-1 and anti-CTLA-4 single-agent or combination immunotherapy [16]. In the present study, we have successfully validated these findings in a homogeneous independent cohort of $N=30$ melanoma patients treated 
A

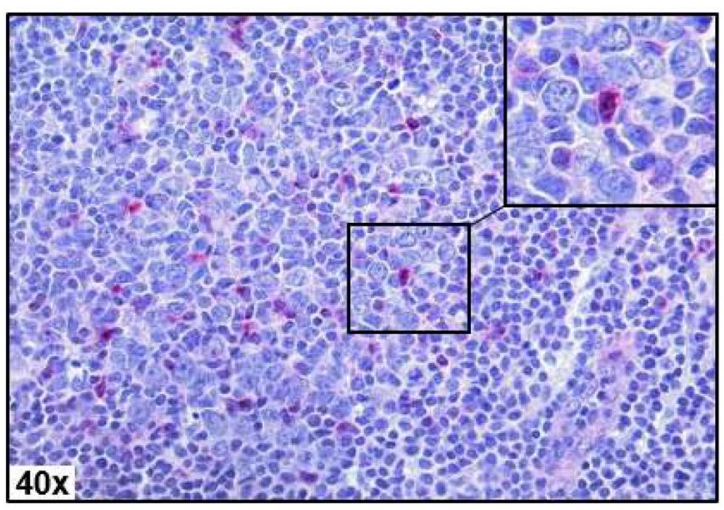

B

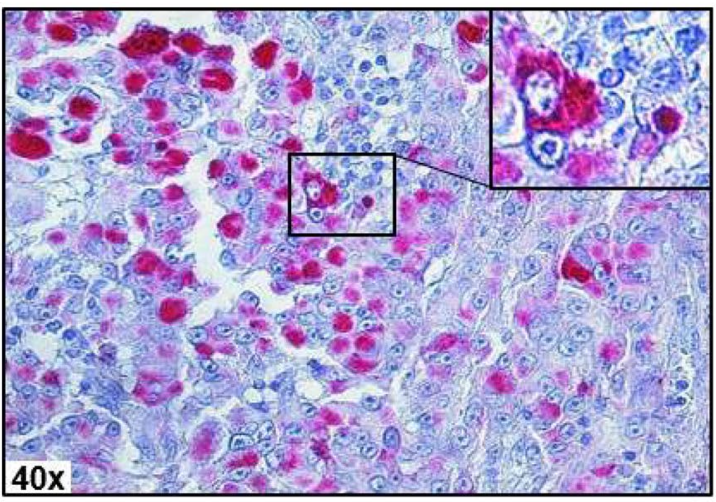

C

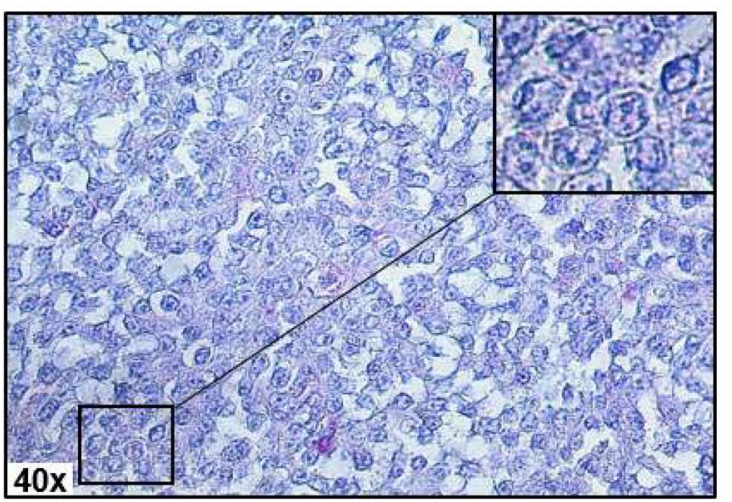

H score: 0

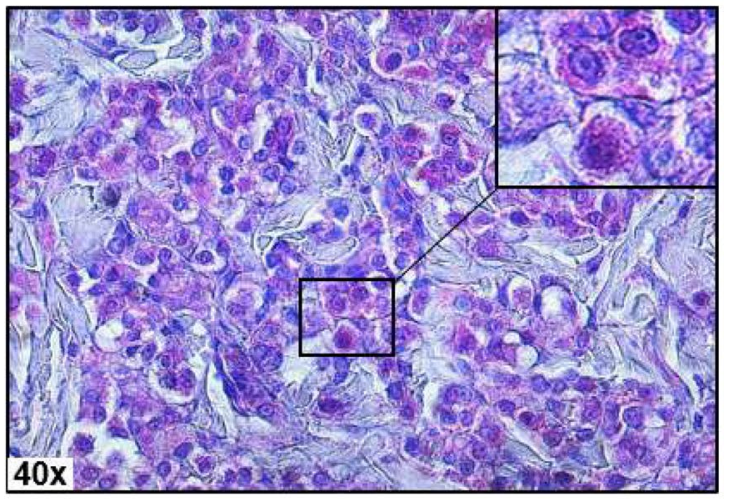

H score: 120

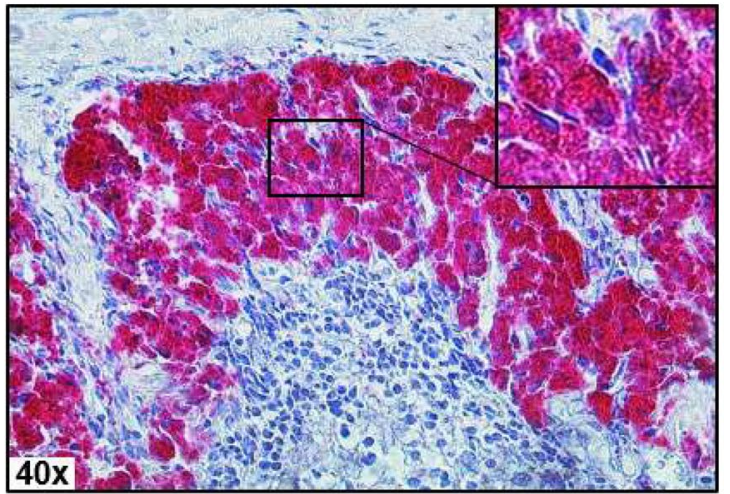

H score: 265

tumor and immune cells (b). Melanomas with mainly CTLA-4-negative, weakly CTLA-4-positive, and strongly CTLA-4-positive tumor cells, respectively, and corresponding $\mathrm{H}$ scores (c)

analyzing high-stage melanoma patients treated with antiCTLA-4 monotherapy [19-21].

CTLA-4 protein was mainly expressed by melanoma cells and by a subset of tumor-infiltrating immune cells. The exact mode of action of anti-CTLA-4 ICB and the role of tumor cell-intrinsic CTLA-4 expression with regard to responsiveness to ICB is still only poorly understood. A study by 
Daud et al. [22] revealed that an increase in the fraction of a certain type of $\mathrm{CD} 8^{+} \mathrm{T}$ cells with high expression of PD-1 and CTLA-4 (PD- $1^{\text {high }} /$ CTLA- $4^{\text {high }}$ ) in metastatic melanoma correlates with response to anti-PD-1 treatment. Further analysis indicated a partially exhausted phenotype (PD-1 ${ }^{\text {high }} /$ CTLA $-4^{\text {high }}$ ) suggesting that ICB might invigorate particularly PD- $1^{\text {high} / C T L A-~} 4{ }^{\text {high }} \mathrm{CD} 8^{+}$T cells. On tumor cells, expression of PD-L1 could be observed in multiple malignancies and seems to be associated with abundance of TILs [5] and interferon $\gamma$ response signatures [23]. CTLA-4 protein expression by melanoma cells could be shown by Mo et al. [24]. The role of CTLA-4 in tumor cells, however, is barely understood. In the same study, a survival benefit under immunotherapy was suggested in patients whose tumors showed an interferon $\gamma$ signature that was associated with a high expression of CTLA-4 mRNA. The predictive value of CTLA-4 mRNA and protein expression in melanoma remains ambiguous.

Our study suggests a correlation between the presence of tumor-infiltrating lymphocytes and CTLA4 promoter methylation. These findings are consistent with our recently published methylation data from peripheral blood leukocytes. Among different subtypes of immune cells, $\mathrm{CD} 4^{+}$and $\mathrm{CD} 8^{+}$ $\mathrm{T}$ cells, which play a key role in tumor control, were associated with a significantly lower methylation level in the CTLA4 gene [25]. Consequently, melanoma samples with a lower CTLA4 promoter methylation level might exhibit a certain immune cell infiltration pattern contributing to response to anti-CTLA-4 immunotherapy. Moreover, our study showed no correlation between CTLA4 promoter methylation and CTLA-4 protein expression in melanoma. Since we have previously shown a strong inverse correlation between methylation and mRNA expression, this finding needs further investigation, ideally including additional CpG sites since CTLA4 DNA methylation highly depends on the sequence context $[16,25]$. Hypothetical explanations are expression of different CTLA-4 isoforms, posttranslational modifications (e.g., glycosylation), inter- and intratumorally different CTLA-4 turnover rates, and the small sample size. We performed IHC CTLA-4 analysis using a CE IVD-certified monoclonal anti-CTLA-4 antibody intended for in vitro diagnostic. Developed in compliance with Invitro Diagnostic Directive (IVDD)/Directive 98/79/EC, we expect a high specificity of this antibody which we confirmed using tonsillar tissue as positive control. However, the epitope of this antibody has not been disclosed by the manufacturer, and results regarding the effect of posttranslational modifications on the antibody binding affinity are not publicly available. Four different CTLA-4 protein isoforms can be generated by alternative splicing: full-length CTLA-4 (exons 1- 4), soluble CTLA-4 (without exon 3), ligand-independent CTLA-4 (without exon 2, murine only), and an isoform using only exons 1 and 4 [26]. CTLA-4 is primarily an intracellular antigen and its surface expression is characterized by restricted trafficking to the cell surface and a rapid internalization. Intracellular CTLA-4 is associated with trans-Golgi network and is found in endosomes, secretory granules, and lysosomal vesicles. The regulation of CTLA-4 surface expression might be influenced by glycosylation [26]. The complex spatial distribution, alternative exon usage, and glycosylation might impair an accurate IHC detection. In concordance, no reports have been published that suggest CTLA-4 protein expression as a predictive biomarker for anti-CTLA-4 ICB which is in line with our findings.

The main limitations of our study are the small sample size, the heterogeneity of included patients regarding clinical and molecular features (cutaneous/non-cutaneous, BRAFmutated/wild-type, sample origin [primary tumor/distant metastases/lymph node metastases], pre-treatment), and the analysis of only a limited number of $\mathrm{CpG}$ sites that are targeted by the used qMSP assay.

Our study directly links DNA promoter methylation of an immune checkpoint to response to blockade of this particular immune checkpoint. Despite the limited clinical significance of the outdated anti-CTLA-4 ICB monotherapy, our results are of importance for mechanism-driven biomarker strategies in the context of immunotherapies, especially since the protein expression of immune checkpoints only insufficiently predicts response to ICB.

\section{Availability of data and material}

The data that support the findings of this study are available from the corresponding author upon reasonable request.

Acknowledgements We thank the BioBank Bonn of the Bonn University Medical Faculty, the University Hospital Bonn, and the Deutsche Forschungsgemeinschaft (DFG) for the support of this study.

Author contributions DD and JL were involved in the study design and concept. SF, JL, and DD drafted the manuscript. DD and SF performed the statistical analysis. RZ and SF conducted the experiments. DN, $\mathrm{CP}, \mathrm{PB}, \mathrm{GHG}, \mathrm{TP}, \mathrm{GK}$, and JL provided patients' samples and clinical data. DN, CP, PB, GHG, SS, TP, GK, and FB revised the manuscript for critical intellectual content. All the authors read and approved the final version of the manuscript.

Funding Open Access funding enabled and organized by Projekt DEAL. SF received funding from the University Hospital Bonn BONFOR program (O-105.0069). DN was funded in part by DFG Cluster of Excellence ImmunoSensation (EXC 1023). The study was in part funded by Qiagen.

\section{Compliance with ethical standards}

Conflict of interest JS is a consultant/advisory board member and received speaker's honoraria from Novartis, BMS, MSD, and Roche. 
DN has received speakers' honoraria or travel expense reimbursements from the following companies: BMS, Novartis, GSK, Celgene, and MSD. JL is a consultant/advisory board member and received speaker's honoraria from Novartis, BMS, MSD, and Roche. DD owns patents and patent applications on biomarker technologies and methylation of immune checkpoint genes as predictive and prognostic biomarkers. The patents are licensed to Qiagen GmbH (Hilden, Germany). DD is a consultant for AJ Innuscreen GmbH (Berlin, Germany) and receives royalties from product sales. The other authors have declared that no conflict of interest exists.

Ethics approval Our study was approved by the Institutional Review Board (IRB) of the University Hospital Bonn (vote no. 187/16).

Consent to participate The ethics committee waived the requirement to obtain informed consent.

Open Access This article is licensed under a Creative Commons Attribution 4.0 International License, which permits use, sharing, adaptation, distribution and reproduction in any medium or format, as long as you give appropriate credit to the original author(s) and the source, provide a link to the Creative Commons licence, and indicate if changes were made. The images or other third party material in this article are included in the article's Creative Commons licence, unless indicated otherwise in a credit line to the material. If material is not included in the article's Creative Commons licence and your intended use is not permitted by statutory regulation or exceeds the permitted use, you will need to obtain permission directly from the copyright holder. To view a copy of this licence, visit http://creativecommons.org/licenses/by/4.0/.

\section{References}

1. Hodi FS, O'Day SJ, McDermott DF et al (2010) Improved survival with ipilimumab in patients with metastatic melanoma. $\mathrm{N}$ Engl J Med 363:711-723

2. Larkin J, Chiarion-Sileni V, Gonzales R et al (2019) Five-year survival with combined nivolumab and ipilimumab in advanced melanoma. N Engl J Med 381:1535-1546

3. Price KD, Simutis F, Fletcher A et al (2018) Nonclinical safety evaluation of two distinct second generation variants of antiCTLA4 monoclonal antibody, ipilimumab, in monkeys. Mol Cancer Ther 17(1 Supplement):LB-B33

4. Nishino M, Ramaiya NH, Hatabu H, Hodi FS (2017) Monitoring immune-checkpoint blockade: response evaluation and biomarker development. Nat Rev Clin Oncol 14(11):655-668

5. Tumeh PC, Harview CL, Yearley JH et al (2014) PD-1 blockade induces responses by inhibiting adaptive immune resistance. Nature 515(7528):568-571

6. Snyder A, Makarov V, Merghoub T et al (2014) Genetic basis for clinical response to CTLA-4 blockade in melanoma. N Engl J Med 371(23):2189-2199

7. Nathanson T, Ahuja A, Rubinsteyn A et al (2017) Somatic mutations and neoepitope homology in melanomas treated with CTLA-4 blockade. Cancer Immunol Res 5(1):84-91

8. Jones PA, Issa JP, Baylin S (2016) Targeting the cancer epigenome for therapy. Nat Rev Genet 17(10):630-641

9. Youngblood B, Hale JS, Kissick HT et al (2017) Effector CD8 $\mathrm{T}$ cells dedifferentiate into long-lived memory cells. Nature 552(7685):404-409
10. Ghoneim HE, Fan Y, Moustaki A et al (2017) De novo epigenetic programs inhibit PD-1 blockade-mediated $\mathrm{T}$ cell rejuvenation. Cell 170(1):142-157

11. Ren DR, Kaminski J, Barnitz RA et al (2016) The epigenetic landscape of T cell exhaustion. Science 354(6316):1165-1169

12. Pauken KE, Sammons MA, Odorizzi PM et al (2016) Epigenetic stability of exhausted T cells limits durability of reinvigoration by PD-1 blockade. Science 354(6316):1160-1165

13. Fröhlich A, Loick S, Bawden EG et al (2020) Comprehensive analysis of tumor necrosis factor receptor TNFRSF9 (4-1BB) DNA methylation with regard to molecular and clinicopathological features, immune infiltrates, and response prediction to immunotherapy in melanoma. EBioMedicine 52:102647

14. Fröhlich A, Sirokay J, Fietz S et al (2020) Molecular, clinicopathological, and immune correlates of $L A G 3$ promoter DNA methylation in melanoma. EBioMedicine 59:102962

15. Hoffmann F, Zarbl R, Niebel D et al (2020) Prognostic and predictive value of $P D-L 2$ DNA methylation and mRNA expression in melanoma. Clin Epigenetics 12(1):94

16. Goltz D, Gevensleben H, Vogt TJ et al (2018) CTLA4 methylation predicts response to anti-PD-1 and anti-CTLA-4 immunotherapy in melanoma patients. JCI Insight 3(13):e9679316

17. Lee HH, Wang YN, Xia W et al (2019) Removal of N-Linked glycosylation enhances PD-L1 detection and predicts Anti-PD-1/ PD-L1 therapeutic efficacy. Cancer Cell 36(2):168-178

18. Němejcová K, Tichá I, Bártů M (2019) Comparison of five different scoring methods in the evaluation of inflammatory infiltration (tumor-infiltrating lymphocytes) in superficial spreading and nodular melanoma. Pigment Cell Melanoma Res 32(3):412-423

19. Postow MA, Chesney J, Pavlick AC et al (2015) Nivolumab and Ipilimumab versus Ipilimumab in Untreated Melanoma. N Engl J Med 372(21):2006-2017

20. Petrelli F, Ardito R, Merelli B et al (2019) Prognostic and predictive role of elevated lactate dehydrogenase in patients with melanoma treated with immunotherapy and BRAF inhibitors: a systematic review and meta-analysis. Melanoma Res 29(1):1-12

21. Conforti F, Pala L, Bagnardi V et al (2018) Cancer immunotherapy efficacy and patients' sex: a systematic review and metaanalysis. Lancet Oncol 19(6):737-746

22. Daud AI, Loo K, Pauli ML et al (2016) Tumor immune profiling predicts response to anti-PD-1 therapy in human melanoma. J Clin Invest 126(9):3447-3452

23. Bald T, Landsberg J, Lopez-Ramos D et al (2014) Immune cellpoor melanomas benefit from PD-1 blockade after targeted type I IFN activation. Cancer Discov 4(6):674-687

24. Mo X, Zhang H, Preston $S$ et al (2018) Interferon-y signaling in melanocytes and melanoma cells regulates expression of CTLA-4. Cancer Res 78(2):436-450

25. de Vos L, Grünwald I, Bawden EG et al (2020) The landscape of CD28, CD80, CD86, CTLA4 and ICOS DNA methylation in head and neck squamous cell carcinomas. Epigenetics 15(11):1195-1212

26. Valk E, Rudd CE, Schneider H (2008) CTLA-4 trafficking and surface expression. Trends Immunol 29(6):272-279

Publisher's Note Springer Nature remains neutral with regard to jurisdictional claims in published maps and institutional affiliations. 